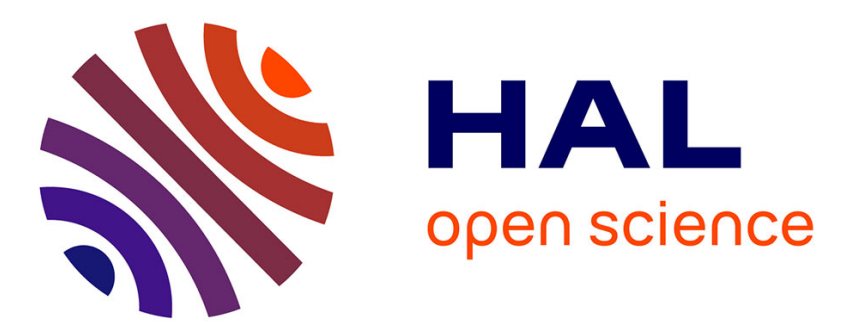

\title{
Dissipative effects on a generation scheme of a W state in an array of coupled Josephson junctions
} R Migliore, M Scala, A Napoli, K Yuasa, H Nakazato, A Messina

\section{To cite this version:}

R Migliore, M Scala, A Napoli, K Yuasa, H Nakazato, et al.. Dissipative effects on a generation scheme of a W state in an array of coupled Josephson junctions. Journal of Physics B: Atomic, Molecular and Optical Physics, 2011, 44 (7), pp.75503. 10.1088/0953-4075/44/7/075503 . hal-00608728

\section{HAL Id: hal-00608728 \\ https://hal.science/hal-00608728}

Submitted on 14 Jul 2011

HAL is a multi-disciplinary open access archive for the deposit and dissemination of scientific research documents, whether they are published or not. The documents may come from teaching and research institutions in France or abroad, or from public or private research centers.
L'archive ouverte pluridisciplinaire HAL, est destinée au dépôt et à la diffusion de documents scientifiques de niveau recherche, publiés ou non, émanant des établissements d'enseignement et de recherche français ou étrangers, des laboratoires publics ou privés. 


\title{
Dissipative effects on a scheme of generation of a $\mathrm{W}$ state in an array of coupled Josephson junctions
}

\author{
R. Migliore \\ Institute of Biophysics, National Research Council, via Ugo La Malfa 153, 90146 \\ Palermo, Italy \\ E-mail: rosanna@fisica.unipa.it
}

\section{Scala}

Dipartimento di Scienze Fisiche ed Astronomiche dell'Università di Palermo, Via Archirafi 36, 90123 Palermo, Italy

E-mail: matteo.scala@fisica.unipa.it

\section{A. Napoli}

Dipartimento di Scienze Fisiche ed Astronomiche dell'Università di Palermo, Via Archirafi 36, 90123 Palermo, Italy

\section{K. Yuasa}

Waseda Institute for Advanced Study, Waseda University, Tokyo 169-8050, Japan

\section{H. Nakazato}

Department of Physics, Waseda University, Okubo 3-4-1, Shinjuku, Tokyo 169-8555, Japan

\section{A. Messina}

Dipartimento di Scienze Fisiche ed Astronomiche dell'Università di Palermo, Via Archirafi 36, 90123 Palermo, Italy

\begin{abstract}
The dynamics of an open quantum system, consisting of three superconducting qubits interacting with independent reservoirs, is investigated to elucidate the effects of the environment on a unitary generation scheme of $\mathrm{W}$ states [Migliore R. et al. (2006) Phys. Rev. B 74, 104503]. To this end a microscopic master equation is constructed and its exact resolution predicts the generation of a Werner-like state instead of the W state. A comparison between our model and a more intuitive phenomenological model is also considered, in order to find the limits of the latter approach in the case of structured reservoirs.
\end{abstract}




\section{Introduction}

The recent progress during the last decade in the context of superconducting solid-state devices, whose system parameters can be controlled in situ to manipulate quantum states coherently, has strengthened the idea that Josephson architectures are among the best candidates both for the search of experimental evidence of nonclassical features at mesoscopic level and for the practical implementation of logic gates and circuits. Superconducting circuits indeed work well, and experiments have demonstrated singlequbit operations $[1,2,3,4,5,6,7]$ with reasonably long coherence times (of the order of $1 \mu \mathrm{s}[8,9])$ and that two-qubit entanglement can be produced $[10,11,12,13,14]$ and detected $[15,16,17]$. A current challenge is the generation of maximally entangled states of more than two solid-state qubits, a powerful resource in quantum information processing and communication. Numerous theoretical protocols for the preparation of such states have been proposed $[18,19,20]$ and very recently also some experimental successes have been achieved. Indeed, tripartite interactions between two phase qubits and a resonant cavity in circuit QED were demonstrated [21], as well as the generation and detection of GHZ-class entanglement between three superconducting phase qubits [22] and three charge qubits [23]. Quite often, the samples were designed with four qubits to allow for future expansion of the multipartite entanglement generation schemes $[21,22]$. All these goals clearly highlight the need of theoretical analyses of the dynamics of superconductive architectures on the basis of more realistic models, including the effects of external environments which are unavoidably coupled to the systems under study.

Indeed, every quantum system is coupled to one or more external environments. Such a coupling may dramatically affect the predictions done under the hypothesis of perfect isolation. When the external environment is included, the dynamics of the open quantum system under study becomes non-unitary, and may suffer dissipation and decoherence. In this context, the open system dynamics is usually described by means of a Markovian master equation. Many master equations for interacting qubits have been recently introduced in the literature, which may turn out to be useful for the study of dissipative effects on multipartite entanglement [24, 25, 26, 27].

Very recently, in the framework of Josephson flux qubits, some of us have proposed a scheme for the generation of multipartite entangled states [19]. This scheme operates in such a way that, exploiting the sequential inductive interaction of each qubit with one of them playing the role of an entanglement mediator, it generates an entangled W state after a finite number of steps without requiring conditional measurements. Two key requirements are the possibilities of both preparing the initial state of the qubits and of switching on and off, at properly selected instant of times, the coupling constant describing the interaction between each qubit and the mediator. The proposed scheme, however, neglects the presence of external degrees of freedom.

In this paper we analyze the theoretical protocol presented in Ref. [19], focussing

in particular our attention on the case of three qubits. In our analysis we take into 
account from the very beginning the coupling between each qubit and the external world by means of a generalization of a previous microscopic master equation for the study of the dissipative dynamics of two coupled qubits [24].

The aim of this paper is twofold. On one hand we investigate the robustness of the theoretical scheme against losses that, generally speaking, waste unitary scheme. On the other hand, the study we develop allows us to give some insights on the general problem of the choice of the effective master equation for the description of open system dynamics. Indeed, while in this paper we propose to study the effects of losses by means of a model microscopically derived, one may wonder what would be the prediction of the phenomenological master equations which are usually adopted in the literature on leaky coupled quantum systems. In particular, while our model involves transitions between stationary states of the whole three-qubit system, the phenomenological approach just describes those between the bare states of the system, i.e., each transition involves one single qubit only. By means of a systematic comparison between the predictions of these two models, we show that, while the models are actually equivalent in the case of flat reservoir spectra, they give very different predictions in the case of structured environments, so that a naive introduction of a phenomenological model can be inappropriate.

The paper is organized as follows. In Sec. II we briefly describe the main features of a tripartite flux qubit system and of a possible coupling scheme which allow us to control at will the interaction between each flux qubit and one of them playing the role of a mediator. We moreover give the microscopic master equation describing this tripartite system. Section III is devoted to the solution of the master equation at zero temperature whereas Sec. IV the analysis of the dynamics starting from appropriate initial conditions exploiting both the microscopic and phenomenological approaches. Concluding remarks are finally summarized in Sec. V.

\section{The physical system and the microscopic Master equation}

Let us begin by briefly illustrating the main features of the tripartite system sketched in Fig. 1, extensively described in Ref. [19]. Two spatially separated flux qubits (named 2 and 3) are connected by tunable flux transformers to a third qubit (M) which plays the role of an entanglement mediator.

Each qubit loop is interrupted by three Josephson junctions. Two of them are characterized by the same Josephson energy $E_{J}$ and the third one possesses a lower Josephson energy $\alpha E_{J}$, with $\alpha<1$. If the operating flux bias $\phi_{x}^{(i)}(i=M, 2,3)$ applied by a coil to each qubit is close to $\phi_{0} / 2, \phi_{0}=h / 2 e$ being the flux quantum, a 3JJ qubit can be described in terms of two quantum states $\left|L^{(i)}\right\rangle$ and $\left|R^{(i)}\right\rangle$ corresponding to a clockwise and a counterclockwise supercurrent circulating in the loop, $\pm I_{p}^{(i)}=2 \pi \alpha E_{J}^{(i)} / \phi_{0}$. In other words, in such conditions it is possible to realize an effective two state system whose Hamiltonian, in the basis of the energy eigenstates $|0\rangle_{i}=\frac{1}{\sqrt{2}}\left[\left|L^{(i)}\right\rangle+\left|R^{(i)}\right\rangle\right]$ and $|1\rangle_{i}=\frac{1}{\sqrt{2}}\left[\left|L^{(i)}\right\rangle-\left|R^{(i)}\right\rangle\right]$, assumes the form $H^{(i)}=\omega_{i} \sigma_{+}^{(i)} \sigma_{-}^{(i)}$ with $\sigma_{+}^{(i)}=|1\rangle_{i}\langle 0|$ and 


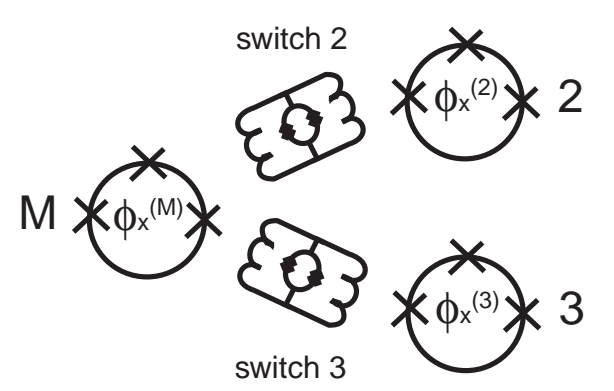

Figure 1. Schematic of the system setup.

$\sigma_{-}^{(i)}=|0\rangle_{i}\langle 1|$. Here the energy splitting $\omega_{i}=\sqrt{\Delta_{i}^{2}+\epsilon_{i}^{2}}$, typically of the order of $5 \div 10 \mathrm{GHz}$, depends on the tunneling frequency $\Delta_{i}$ between the two wells of the qubit potential and on the corresponding asymmetry $\epsilon_{i}=2 I_{p}^{(i)}\left(\phi_{x}^{(i)}-\phi_{0} / 2\right)$. An inductive coupling between the qubits 2 or 3 and the mediator $M$ can be realized via the tunable flux transformer described in Ref. [28]. As shown indeed by M. G. Castellano et al. [28] it is possible to control the flux-transfer function $\mathcal{R}\left(\phi_{c x}^{(i)}\right)$, and therefore the inductivecoupling constants, $g_{M 2}$ and $g_{M 3}$, by properly setting the critical current of the inner dc-SQUID of each transformer via the additional bias fluxes $\phi_{c x}^{(2)}$ and $\phi_{c x}^{(3)}$.

In this way we may turn on and off at will the coupling between each qubit and the mediator, to realize as extensively discussed in Ref. [19], a unitary step-by-step scheme which allows us to generate the following W state of the whole system:

$$
|W\rangle=\frac{1}{\sqrt{3}}(|100\rangle-i|010\rangle-i|001\rangle) \text {. }
$$

Here we want to investigate the effects of dissipation on this generation scheme caused by environments. Let us start by briefly summarizing the scheme for the generation of the state (1) starting from the initial condition $|100\rangle$ of the three qubits, in which only the first qubit is excited, whereas the other two are in their ground states.

Calling $M$ the first qubit and 2 and 3 the other two, the scheme consists of two steps: in the first step the qubit $\mathrm{M}$ interacts for an interval of time $\tau_{1}$ with the qubit 2 and the qubit 3 evolves freely, while in the second step the qubit M interacts for a time $\tau_{2}$ with the qubit 3 and the qubit 2 evolves freely. The Hamiltonian models corresponding to these two steps are respectively:

$$
H_{1 \mathrm{st}}=\sum_{i=M, 2,3} \omega_{i} \sigma_{+}^{(i)} \sigma_{-}^{(i)}+g_{M 2}\left(\sigma_{+}^{(M)} \sigma_{-}^{(2)}+\sigma_{-}^{(M)} \sigma_{+}^{(2)}\right),
$$

and

$$
H_{2 \mathrm{nd}}=\sum_{i=M, 2,3} \omega_{i} \sigma_{+}^{(i)} \sigma_{-}^{(i)}+g_{M 3}\left(\sigma_{+}^{(M)} \sigma_{-}^{(3)}+\sigma_{-}^{(M)} \sigma_{+}^{(3)}\right) .
$$

The explicit expressions for the eigenstates of $H_{1 \mathrm{st}}\left(H_{2 \mathrm{nd}}\right)$ denoted by $|a\rangle \otimes|k\rangle_{3}$, $|b\rangle \otimes|k\rangle_{3},|c\rangle \otimes|k\rangle_{3}$ and $|d\rangle \otimes|k\rangle_{3}\left(\left|a^{\prime}\right\rangle \otimes|k\rangle_{2},\left|b^{\prime}\right\rangle \otimes|k\rangle_{2},\left|c^{\prime}\right\rangle \otimes|k\rangle_{2}\right.$ and $\left.\left|d^{\prime}\right\rangle \otimes|k\rangle_{2}\right)$, where $k=0,1$, and the corresponding eigenvalues are given in the Appendix. During these two steps, the three qubits suffer from environmental perturbations. We assume that 
each qubit interacts with a reservoir which is independent from the others, since the three qubits are spatially separated, and we exploit the results presented in Ref. [24], where a master equation for the evolution of two coupled qubits has been derived from a microscopic Hamiltonian, following the standard approach [29]. More in detail, given the Hamiltonian of a generic quantum system, the microscopically derived master equation, in the Born-Markov and rotating wave approximations, describes jumps occurring between the eigenstates of the Hamiltonian. Moreover in Ref. [24] the reservoirs are assumed to be independent, which is justified in the case of spatially separated Josephson qubits, since the noise in each device is due to impurities belonging to different parts of the whole superconducting circuit.

Our idea is now to use the dissipator appearing in that paper to describe the losses of the pair of coupled qubits both in the first and in the second step, while the dissipation relative to the free $i$-th qubit ( $i$ is equal either to 2 or 3 , depending on the step considered) will be described as usual by terms like $\gamma_{i}\left(\sigma_{-}^{(i)} \rho \sigma_{+}^{(i)}-\frac{1}{2}\left\{\sigma_{+}^{(i)} \sigma_{-}^{(i)}, \rho\right\}\right)$.

More in detail, exploiting the results presented in Ref.[24], in correspondence to the first step we can write:

$$
\dot{\rho}=-i\left[H_{1 \mathrm{st}}, \rho\right]+\mathcal{D}_{M 2}(\rho)+\mathcal{D}_{3}(\rho),
$$

while for the second step the master equation can be given as:

$$
\dot{\rho}=-i\left[H_{2 \mathrm{nd}}, \rho\right]+\mathcal{D}_{M 3}(\rho)+\mathcal{D}_{2}(\rho) .
$$

The two dissipators $\mathcal{D}_{M 2}$ and $\mathcal{D}_{M 3}$ describe the losses of the combined systems of qubits $\mathrm{M}$ and 2, and qubits $\mathrm{M}$ and 3 respectively, and their structure can be directly obtained starting from Ref. [24]. In particular the dissipator in Eq. (4) can be written as:

$$
\begin{aligned}
& \mathcal{D}_{M 2}(\rho)=c_{I}\left(|a\rangle\langle b|\rho(t)| b\rangle\langle a|-\frac{1}{2}\{|b\rangle\langle b|, \rho(t)\}\right) \\
& +c_{I I}\left(|a\rangle\langle c|\rho(t)| c\rangle\langle a|-\frac{1}{2}\{|c\rangle\langle c|, \rho(t)\}\right) \\
& +c_{I}\left(|b\rangle\langle d|\rho(t)| d\rangle\langle b|-\frac{1}{2}\{|d\rangle\langle d|, \rho(t)\}\right) \\
& +c_{I I}\left(|c\rangle\langle d|\rho(t)| d\rangle\langle c|-\frac{1}{2}\{|d\rangle\langle d|, \rho(t)\}\right) \\
& +\bar{c}_{I}\left(|b\rangle\langle a|\rho(t)| a\rangle\langle b|-\frac{1}{2}\{|a\rangle\langle a|, \rho(t)\}\right) \\
& +\bar{c}_{I I}\left(|c\rangle\langle a|\rho(t)| a\rangle\langle c|-\frac{1}{2}\{|a\rangle\langle a|, \rho(t)\}\right) \\
& +\bar{c}_{I}\left(|d\rangle\langle b|\rho(t)| b\rangle\langle d|-\frac{1}{2}\{|b\rangle\langle b|, \rho(t)\}\right) \\
& +\bar{c}_{I I}\left(|d\rangle\langle c|\rho(t)| c\rangle\langle d|-\frac{1}{2}\{|c\rangle\langle c|, \rho(t)\}\right) \\
& +c_{c r, I}(|a\rangle\langle b|\rho(t)| d\rangle\langle c|+| c\rangle\langle d|\rho(t)| b\rangle\langle a|)
\end{aligned}
$$


Dissipative effects on a scheme of generation of a W state in an array of coupled Josephson junctions6

$$
\begin{aligned}
& +c_{c r, I I}(|a\rangle\langle c|\rho(t)| d\rangle\langle b|+| b\rangle\langle d|\rho(t)| c\rangle\langle a|) \\
& +\bar{c}_{c r, I}(|d\rangle\langle c|\rho(t)| a\rangle\langle b|+| b\rangle\langle a|\rho(t)| c\rangle\langle d|) \\
& +\bar{c}_{c r, I I}(|d\rangle\langle b|\rho(t)| a\rangle\langle c|+| c\rangle\langle a|\rho(t)| b\rangle\langle d|),
\end{aligned}
$$

where $|a\rangle,|b\rangle,|c\rangle$ and $|d\rangle$ are given by Eqs. (A.1) in the Appendix. The decay rates appearing in Eq. (6) are defined as:

$$
\begin{aligned}
& c_{I}=\gamma_{I, M} \cos ^{2} \frac{\theta}{2}+\gamma_{I, 2} \sin ^{2} \frac{\theta}{2}, \\
& c_{I I}=\gamma_{I I, M} \sin ^{2} \frac{\theta}{2}+\gamma_{I I, 2} \cos ^{2} \frac{\theta}{2}, \\
& c_{c r, I}=\gamma_{I, M} \cos ^{2} \frac{\theta}{2}-\gamma_{I, 2} \sin ^{2} \frac{\theta}{2}, \\
& c_{c r, I I}=-\gamma_{I I, M} \sin ^{2} \frac{\theta}{2}+\gamma_{I I, 2} \cos ^{2} \frac{\theta}{2},
\end{aligned}
$$

where for brevity we have put $\gamma_{I, j}=\gamma_{j}\left(\omega_{I}\right)$ and $\gamma_{I I, j}=\gamma_{j}\left(\omega_{I I}\right)$, with

$$
\omega_{I}=\frac{1}{2}\left(\omega_{M}+\omega_{2}\right)-\frac{1}{2} \sqrt{\left(\omega_{2}-\omega_{M}\right)^{2}+g_{M 2}^{2}},
$$

the Bohr frequency relative to the transitions $|d\rangle \rightarrow|c\rangle$ and $|b\rangle \rightarrow|a\rangle$ and

$$
\omega_{I I}=\frac{1}{2}\left(\omega_{M}+\omega_{2}\right)+\frac{1}{2} \sqrt{\left(\omega_{2}-\omega_{M}\right)^{2}+g_{M 2}^{2}}
$$

the Bohr frequency relative to the transitions $|c\rangle \rightarrow|a\rangle$ and $|d\rangle \rightarrow|b\rangle$. The functions $\gamma_{j}(\omega)$ are the Fourier transforms of the bath correlation functions of the $j$ th reservoir (with $j=\mathrm{M}, 2,3$ ) [24], and are related to the $j$-th reservoir spectral density $J_{j}(\omega)$ through the relation:

$$
\gamma_{j}(\omega)=J_{j}(\omega)\left[1+N_{j}(\omega)\right]
$$

with $N_{j}(\omega)$ being the average number of photons in the mode of frequency $\omega$. The excitation rates $\bar{c}_{I}, \bar{c}_{I I}, \bar{c}_{c r, I}$ and $\bar{c}_{c r, I I}$ can be directly obtained from Eqs. (7)-(10) correspondingly substituting $\gamma_{I, j}$ by $\bar{\gamma}_{I, j}=\gamma_{I, j} \mathrm{e}^{-\beta_{j} \omega_{I}}$ and $\gamma_{I I, j}$ by $\bar{\gamma}_{I I, j}=\gamma_{I I, j} \mathrm{e}^{-\beta_{j} \omega_{I I}}$, with $\beta_{j}$ the inverse temperature of the $j$-th reservoir.

In the same way, the dissipator in Eq. (5) is given by:

$$
\begin{aligned}
& \mathcal{D}_{M 3}(\rho)=c_{I}^{\prime}\left(\left|a^{\prime}\right\rangle\left\langle b^{\prime}|\rho(t)| b^{\prime}\right\rangle\left\langle a^{\prime}\right|-\frac{1}{2}\left\{\left|b^{\prime}\right\rangle\left\langle b^{\prime}\right|, \rho(t)\right\}\right) \\
& +c_{I I}^{\prime}\left(\left|a^{\prime}\right\rangle\left\langle c^{\prime}|\rho(t)| c^{\prime \prime}\right\rangle\langle a|-\frac{1}{2}\left\{\left|c^{\prime}\right\rangle\left\langle c^{\prime}\right|, \rho(t)\right\}\right) \\
& +c_{I}^{\prime}\left(\left|b^{\prime}\right\rangle\left\langle d^{\prime}|\rho(t)| d^{\prime}\right\rangle\left\langle b^{\prime}\right|-\frac{1}{2}\left\{\left|d^{\prime}\right\rangle\left\langle d^{\prime}\right|, \rho(t)\right\}\right) \\
& +c_{I I}^{\prime}\left(\left|c^{\prime}\right\rangle\left\langle d^{\prime}|\rho(t)| d^{\prime}\right\rangle\left\langle c^{\prime}\right|-\frac{1}{2}\left\{\left|d^{\prime}\right\rangle\left\langle d^{\prime}\right|, \rho(t)\right\}\right) \\
& +\bar{c}_{I}^{\prime}\left(\left|b^{\prime}\right\rangle\left\langle a^{\prime}|\rho(t)| a^{\prime}\right\rangle\left\langle b^{\prime}\right|-\frac{1}{2}\left\{\left|a^{\prime}\right\rangle\left\langle a^{\prime}\right|, \rho(t)\right\}\right)
\end{aligned}
$$


Dissipative effects on a scheme of generation of a W state in an array of coupled Josephson junctions 7

$$
\begin{aligned}
& +\bar{c}_{I I}^{\prime}\left(\left|c^{\prime}\right\rangle\left\langle a^{\prime}|\rho(t)| a^{\prime}\right\rangle\left\langle c^{\prime}\right|-\frac{1}{2}\left\{\left|a^{\prime}\right\rangle\left\langle a^{\prime}\right|, \rho(t)\right\}\right) \\
& +\bar{c}_{I}^{\prime}\left(\left|d^{\prime}\right\rangle\left\langle b^{\prime}|\rho(t)| b^{\prime}\right\rangle\left\langle d^{\prime}\right|-\frac{1}{2}\left\{\left|b^{\prime}\right\rangle\left\langle b^{\prime}\right|, \rho(t)\right\}\right) \\
& +\bar{c}_{I I}^{\prime}\left(\left|d^{\prime}\right\rangle\left\langle c^{\prime}|\rho(t)| c^{\prime}\right\rangle\left\langle d^{\prime}\right|-\frac{1}{2}\left\{\left|c^{\prime}\right\rangle\left\langle c^{\prime}\right|, \rho(t)\right\}\right) \\
& +c_{c r, I}^{\prime}\left(\left|a^{\prime}\right\rangle\left\langle b^{\prime}|\rho(t)| d^{\prime}\right\rangle\left\langle c^{\prime}|+| c^{\prime}\right\rangle\left\langle d^{\prime}|\rho(t)| b^{\prime}\right\rangle\left\langle a^{\prime}\right|\right) \\
& +c_{c r, I I}^{\prime}\left(\left|a^{\prime}\right\rangle\left\langle c^{\prime}|\rho(t)| d^{\prime}\right\rangle\left\langle b^{\prime}|+| b^{\prime}\right\rangle\left\langle d^{\prime}|\rho(t)| c^{\prime}\right\rangle\left\langle a^{\prime}\right|\right) \\
& +\bar{c}_{c r, I}^{\prime}\left(\left|d^{\prime}\right\rangle\left\langle c^{\prime}|\rho(t)| a^{\prime}\right\rangle\left\langle b^{\prime}|+| b^{\prime}\right\rangle\left\langle a^{\prime}|\rho(t)| c^{\prime}\right\rangle\left\langle d^{\prime}\right|\right) \\
& +\bar{c}_{c r, I I}^{\prime}\left(\left|d^{\prime}\right\rangle\left\langle b^{\prime}|\rho(t)| a^{\prime}\right\rangle\left\langle c^{\prime}|+| c^{\prime}\right\rangle\left\langle a^{\prime}|\rho(t)| b^{\prime}\right\rangle\left\langle d^{\prime}\right|\right),
\end{aligned}
$$

where $\left|a^{\prime}\right\rangle,\left|b^{\prime}\right\rangle,\left|c^{\prime}\right\rangle$ and $\left|d^{\prime}\right\rangle$ are given by Eqs. (A.4) and:

$$
\begin{aligned}
& c_{I}^{\prime}=\gamma_{I, M}^{\prime} \cos ^{2} \frac{\theta^{\prime}}{2}+\gamma_{I, 2}^{\prime} \sin ^{2} \frac{\theta^{\prime}}{2}, \\
& c_{I I}^{\prime}=\gamma_{I I, M}^{\prime} \sin ^{2} \frac{\theta^{\prime}}{2}+\gamma_{I I, 2}^{\prime} \cos ^{2} \frac{\theta^{\prime}}{2}, \\
& c_{c r, I}^{\prime}=\gamma_{I, M}^{\prime} \cos ^{2} \frac{\theta^{\prime}}{2}-\gamma_{I, 2}^{\prime} \sin ^{2} \frac{\theta^{\prime}}{2}, \\
& c_{c r, I I}^{\prime}=-\gamma_{I I, M}^{\prime} \sin ^{2} \frac{\theta^{\prime}}{2}+\gamma_{I I, 2}^{\prime} \cos ^{2} \frac{\theta^{\prime}}{2} .
\end{aligned}
$$

The excitation rates $\bar{c}_{I}^{\prime}, \bar{c}_{I I}^{\prime}, \bar{c}_{c r, I}^{\prime}$ and $\bar{c}_{c r, I I}^{\prime}$ can be directly obtained from Eqs. (15)-(18) correspondingly substituting $\gamma_{I, j}^{\prime}$ by $\bar{\gamma}_{I, j}^{\prime}=\gamma_{I, j}^{\prime} \mathrm{e}^{-\beta_{j} \omega_{I}^{\prime}}$ and $\gamma_{I I, j}^{\prime}$ by $\bar{\gamma}_{I I, j}^{\prime}=\gamma_{I I, j}^{\prime} \mathrm{e}^{-\beta_{j} \omega_{I I}^{\prime}}$, where

$$
\begin{aligned}
\omega_{I}^{\prime} & =\frac{1}{2}\left(\omega_{M}+\omega_{3}\right)-\frac{1}{2} \sqrt{\left(\omega_{3}-\omega_{M}\right)^{2}+g_{M 3}^{2}}, \\
\omega_{I I}^{\prime} & =\frac{1}{2}\left(\omega_{M}+\omega_{3}\right)+\frac{1}{2} \sqrt{\left(\omega_{3}-\omega_{M}\right)^{2}+g_{M 3}^{2}} .
\end{aligned}
$$

Finally, we define the single qubit dissipators appearing in Eqs. (4) and (5)

$$
\begin{aligned}
\mathcal{D}_{3}(\rho) & =\gamma_{3}\left(\sigma_{-}^{(3)} \rho \sigma_{+}^{(3)}-\frac{1}{2}\left\{\sigma_{+}^{(3)} \sigma_{-}^{(3)}, \rho\right\}\right) \\
& +\bar{\gamma}_{3}\left(\sigma_{+}^{(3)} \rho \sigma_{-}^{(3)}-\frac{1}{2}\left\{\sigma_{-}^{(3)} \sigma_{+}^{(3)}, \rho\right\}\right), \\
\mathcal{D}_{2}(\rho) & =\gamma_{2}\left(\sigma_{-}^{(2)} \rho \sigma_{+}^{(2)}-\frac{1}{2}\left\{\sigma_{+}^{(2)} \sigma_{-}^{(2)}, \rho\right\}\right) \\
& +\bar{\gamma}_{2}\left(\sigma_{+}^{(2)} \rho \sigma_{-}^{(2)}-\frac{1}{2}\left\{\sigma_{-}^{(2)} \sigma_{+}^{(2)}, \rho\right\}\right),
\end{aligned}
$$

where $\gamma_{3}=\gamma_{3}\left(\omega_{3}\right)$ and $\gamma_{2}=\gamma_{2}\left(\omega_{2}\right)\left(\bar{\gamma}_{3}=\gamma_{3} \mathrm{e}^{-\beta_{j} \omega_{3}}\right.$ and $\left.\bar{\gamma}_{2}=\gamma_{2} \mathrm{e}^{-\beta_{j} \omega_{2}}\right)$ are the decay (excitation) rates of the qubits 3 and 2, which evolve freely during the first and second step, respectively. 
Having at our disposal the master equation microscopically derived, we can analyze the dynamics of the system putting into evidence the effects of the presence of the external world on the generation of $\mathrm{W}$ states. In addition, the knowledge of such a master equation gives us the possibility to test the validity of phenomenological master equations generally used to describe physical systems like the one examined in the present paper. A phenomenological approach would suggest us to adopt the following equation to describe the dynamics of the system under scrutiny

$$
\begin{aligned}
\dot{\rho} & =-i[H, \rho]+\sum_{i=M, 2,3} \gamma_{i}\left(\sigma_{-}^{(i)} \rho \sigma_{+}^{(i)}-\frac{1}{2}\left\{\sigma_{+}^{(i)} \sigma_{-}^{(i)}, \rho\right\}\right) \\
& +\sum_{i=M, 2,3} \bar{\gamma}_{i}\left(\sigma_{+}^{(i)} \rho \sigma_{-}^{(i)}-\frac{1}{2}\left\{\sigma_{-}^{(i)} \sigma_{+}^{(i)}, \rho\right\}\right)
\end{aligned}
$$

where $H$ is equal to either $H_{1 \text { st }}$ or $H_{2 \text { nd }}$ depending on the step considered, while the dissipator is the same for both steps. In what follows we refer to Eq. (23) as the phenomenological master equation.

\section{Solution of the microscopic model at zero temperature}

From now on, we will call a microscopic model the set of master equations (4) and (5), while we will call a phenomenological model the master equation (23).

Let us start with the microscopic model. We will focus on the zero-temperature case, i.e., we will put all the excitation rates equal to zero. The initial state we consider is $|100\rangle$, which, in terms of the eigenstates of the first step corresponds to a density operator where the only nonzero matrix elements are between $|b 0\rangle$ and $|c 0\rangle$

$$
\begin{aligned}
|100\rangle\langle 100| & =\left(\cos \frac{\theta}{2}|b\rangle+\sin \frac{\theta}{2}|c\rangle\right)\left(\cos \frac{\theta}{2}\left\langle b\left|+\sin \frac{\theta}{2}\langle c|\right) \otimes \mid 0\right\rangle_{3}\langle 0|\right. \\
& =\left(\cos \frac{\theta}{2}|b 0\rangle+\sin \frac{\theta}{2}|c 0\rangle\right)\left(\cos \frac{\theta}{2}\langle b 0|+\sin \frac{\theta}{2}\langle c 0|\right) .
\end{aligned}
$$

Thus the only differential equations of interest, derivable from the master equation (4), are the following ones:

$$
\begin{aligned}
& \dot{\rho}_{c 0, c 0}=\gamma_{3} \rho_{c 1, c 1}-c_{I I} \rho_{c 0, c 0}, \\
& \dot{\rho}_{c 1, c 1}=-\left(\gamma_{3}+c_{I I}\right) \rho_{c 1, c 1}, \\
& \dot{\rho}_{b 0, b 0}=\gamma_{3} \rho_{b 1, b 1}-c_{I} \rho_{b 0, b 0}, \\
& \dot{\rho}_{b 1, b 1}=-\left(\gamma_{3}+c_{I}\right) \rho_{b 1, b 1}, \\
& \dot{\rho}_{a 0, a 0}=\gamma_{3} \rho_{a 1, a 1}+c_{I} \rho_{b 0, b 0}+c_{I I} \rho_{c 0, c 0}, \\
& \dot{\rho}_{a 1, a 1}=-\gamma_{3} \rho_{a 1, a 1}+c_{I} \rho_{b 1, b 1}+c_{I I} \rho_{c 1, c 1},
\end{aligned}
$$


Dissipative effects on a scheme of generation of a $W$ state in an array of coupled Josephson junctions 9

$$
\begin{aligned}
& \dot{\rho}_{b 0, c 0}=\left(i \omega_{c b}-\frac{c_{I}+c_{I I}}{2}\right) \rho_{b 0, c 0}+\gamma_{3} \rho_{b 1, c 1}, \\
& \dot{\rho}_{b 1, c 1}=\left(i \omega_{c b}-\gamma_{3}-\frac{c_{I}+c_{I I}}{2}\right) \rho_{b 1, c 1},
\end{aligned}
$$

where $\omega_{c b}=E_{c}-E_{b}$.

Solving these equations, we are able to demonstrate that at $t=\tau_{1}$, namely at the end of the first step, the state of the system reads as

$$
\begin{aligned}
\rho\left(\tau_{1}\right) & =\rho_{a 0, a 0}\left(\tau_{1}\right)|a 0\rangle\left\langle a 0\left|+\rho_{b 0, b 0}\left(\tau_{1}\right)\right| b 0\right\rangle\left\langle b 0\left|+\rho_{c 0, c 0}\left(\tau_{1}\right)\right| c 0\right\rangle\langle c 0| \\
& +\rho_{b 0, c 0}\left(\tau_{1}\right)|b 0\rangle\left\langle c 0\left|+\rho_{b 0, c 0}^{*}\left(\tau_{1}\right)\right| c 0\right\rangle\langle b 0|,
\end{aligned}
$$

where

$$
\begin{aligned}
& \rho_{c 0, c 0}\left(\tau_{1}\right)=\rho_{c 0, c 0}(0) \mathrm{e}^{-c_{I I} \tau_{1}}, \\
& \rho_{b 0, b 0}\left(\tau_{1}\right)=\rho_{b 0, b 0}(0) \mathrm{e}^{-c_{I} \tau_{1}}, \\
& \rho_{a 0, a 0}\left(\tau_{1}\right)=1-\rho_{b 0, b 0}(0) \mathrm{e}^{-c_{I} \tau_{1}}-\rho_{c 0, c 0}(0) \mathrm{e}^{-c_{I I} \tau_{1}}, \\
& \rho_{b 0, c 0}\left(\tau_{1}\right)=\rho_{b 0, c 0}(0) \mathrm{e}^{\left(i \omega_{c b}-\frac{c_{I}+c_{I I}}{2}\right) \tau_{1}} .
\end{aligned}
$$

The state described by Eq. (29) represents the initial condition for step 2. In the basis of the eigenstates of the Hamiltonian (3), it reads:

$$
\begin{aligned}
\rho\left(\tau_{1}\right) & =\rho_{a^{\prime} 0, a^{\prime} 0}\left(\tau_{1}\right)\left|a^{\prime} 0\right\rangle\left\langle a^{\prime} 0\left|+\rho_{b^{\prime} 0, b^{\prime} 0}\left(\tau_{1}\right)\right| b^{\prime} 0\right\rangle\left\langle b^{\prime} 0\left|+\rho_{c^{\prime} 0, c^{\prime} 0}\left(\tau_{1}\right)\right| c^{\prime} 0\right\rangle\left\langle c^{\prime} 0\right| \\
& +\rho_{a^{\prime} 1, a^{\prime} 1}\left(\tau_{1}\right)\left|a^{\prime} 1\right\rangle\left\langle a^{\prime} 1\left|+\rho_{b^{\prime} 0, c^{\prime} 0}\left(\tau_{1}\right)\right| b^{\prime} 0\right\rangle\left\langle c^{\prime} 0\left|+\rho_{b^{\prime} 0, c^{\prime} 0}^{*}\left(\tau_{1}\right)\right| c^{\prime} 0\right\rangle\left\langle b^{\prime} 0\right| \\
& +\rho_{b^{\prime} 0, a^{\prime} 1}\left(\tau_{1}\right)\left|b^{\prime} 0\right\rangle\left\langle a^{\prime} 1\left|+\rho_{b^{\prime} 0, a^{\prime} 1}^{*}\left(\tau_{1}\right)\right| a^{\prime} 1\right\rangle\left\langle b^{\prime} 0\left|+\rho_{c^{\prime} 0, a^{\prime} 1}\left(\tau_{1}\right)\right| c^{\prime} 0\right\rangle\left\langle a^{\prime} 1\right| \\
& +\rho_{c^{\prime} 0, a^{\prime} 1}^{*}\left(\tau_{1}\right)\left|a^{\prime} 1\right\rangle\left\langle c^{\prime} 0\right|,
\end{aligned}
$$

where

$$
\begin{aligned}
\rho_{a^{\prime} 1, a^{\prime} 1}\left(\tau_{1}\right) & =\rho_{b 0, b 0}\left(\tau_{1}\right) \sin ^{2} \frac{\theta}{2}+\rho_{c 0, c 0}\left(\tau_{1}\right) \cos ^{2} \frac{\theta}{2} \\
& -2 \operatorname{Re}\left[\rho_{b 0, c 0}\left(\tau_{1}\right)\right] \sin \frac{\theta}{2} \cos \frac{\theta}{2} \\
\rho_{c^{\prime} 0, c^{\prime} 0}\left(\tau_{1}\right) & =\rho_{b 0, b 0}\left(\tau_{1}\right) \cos ^{2} \frac{\theta}{2} \sin ^{2} \frac{\theta^{\prime}}{2}+\rho_{c 0, c 0}\left(\tau_{1}\right) \sin ^{2} \frac{\theta}{2} \sin ^{2} \frac{\theta^{\prime}}{2} \\
& +2 \operatorname{Re}\left[\rho_{b 0, c 0}\left(\tau_{1}\right)\right] \sin \frac{\theta}{2} \cos \frac{\theta}{2} \sin ^{2} \frac{\theta^{\prime}}{2}, \\
\rho_{b^{\prime} 0, b^{\prime} 0}\left(\tau_{1}\right) & =\rho_{b 0, b 0}\left(\tau_{1}\right) \cos ^{2} \frac{\theta}{2} \cos ^{2} \frac{\theta^{\prime}}{2}+\rho_{c 0, c 0}\left(\tau_{1}\right) \sin ^{2} \frac{\theta}{2} \cos ^{2} \frac{\theta^{\prime}}{2} \\
& +2 \operatorname{Re}\left[\rho_{b 0, c 0}\left(\tau_{1}\right)\right] \sin \frac{\theta}{2} \cos \frac{\theta}{2} \cos ^{2} \frac{\theta^{\prime}}{2}, \\
\rho_{a^{\prime} 0, a^{\prime} 0}\left(\tau_{1}\right) & =\rho_{a 0, a 0}\left(\tau_{1}\right), \\
\rho_{b^{\prime} 0, c^{\prime} 0}\left(\tau_{1}\right) & =\left(\rho_{b 0, b 0}\left(\tau_{1}\right) \cos ^{2} \frac{\theta}{2}+\rho_{c 0, c 0}\left(\tau_{1}\right) \sin ^{2} \frac{\theta}{2}\right. \\
& \left.+2 \operatorname{Re}\left[\rho_{b 0, c 0}\left(\tau_{1}\right)\right] \sin \frac{\theta}{2} \cos \frac{\theta}{2}\right) \sin ^{\frac{\theta^{\prime}}{2}} \cos \frac{\theta^{\prime}}{2},
\end{aligned}
$$


Dissipative effects on a scheme of generation of a $W$ state in an array of coupled Josephson junctions10

$$
\begin{aligned}
\rho_{b^{\prime} 0, a^{\prime} 1}\left(\tau_{1}\right) & =\left[-\rho_{b 0, b 0}\left(\tau_{1}\right)+\rho_{c 0, c 0}\left(\tau_{1}\right)\right] \sin \frac{\theta}{2} \cos \frac{\theta}{2} \cos \frac{\theta^{\prime}}{2} \\
& +\rho_{b 0, c 0}\left(\tau_{1}\right) \cos ^{2} \frac{\theta}{2} \cos \frac{\theta^{\prime}}{2}-\rho_{b 0, c 0}^{*}\left(\tau_{1}\right) \sin ^{2} \frac{\theta}{2} \cos \frac{\theta^{\prime}}{2}, \\
\rho_{c^{\prime} 0, a^{\prime} 1}\left(\tau_{1}\right) & =\left[-\rho_{b 0, b 0}\left(\tau_{1}\right)+\rho_{c 0, c 0}\left(\tau_{1}\right)\right] \sin \frac{\theta}{2} \cos \frac{\theta}{2} \sin \frac{\theta^{\prime}}{2} \\
& +\rho_{b 0, c 0}\left(\tau_{1}\right) \cos ^{2} \frac{\theta}{2} \sin \frac{\theta^{\prime}}{2}-\rho_{b 0, c 0}^{*}\left(\tau_{1}\right) \sin ^{2} \frac{\theta}{2} \sin \frac{\theta^{\prime}}{2} .
\end{aligned}
$$

Starting from this initial condition, it is straightforward to see that the only differential equations of interest, derivable from the master equation (5), describing the dynamics of the system during the second step at $T=0 \mathrm{~K}$, are

$$
\begin{aligned}
& \dot{\rho}_{c^{\prime} 0, c^{\prime} 0}=\gamma_{2} \rho_{c^{\prime} 1, c^{\prime} 1}-c_{I I}^{\prime} \rho_{c^{\prime} 0, c^{\prime} 0}, \\
& \dot{\rho}_{c^{\prime} 1, c^{\prime} 1}=-\left(\gamma_{2}+c_{I I}^{\prime}\right) \rho_{c^{\prime} 1, c^{\prime} 1}, \\
& \dot{\rho}_{b^{\prime} 0, b^{\prime} 0}=\gamma_{2} \rho_{b^{\prime} 1, b^{\prime} 1}-c_{I}^{\prime} \rho_{b^{\prime} 0, b^{\prime} 0}, \\
& \dot{\rho}_{b^{\prime} 1, b^{\prime} 1}=-\left(\gamma_{2}+c_{I}^{\prime}\right) \rho_{b^{\prime} 1, b^{\prime} 1}, \\
& \dot{\rho}_{a^{\prime} 0, a^{\prime} 0}=\gamma_{2} \rho_{a^{\prime} 1, a^{\prime} 1}+c_{I}^{\prime} \rho_{b^{\prime} 0, b^{\prime} 0}+c_{I I}^{\prime} \rho_{c^{\prime} 0, c^{\prime} 0}, \\
& \dot{\rho}_{a^{\prime} 1, a^{\prime} 1}=-\gamma_{2} \rho_{a^{\prime} 1, a^{\prime} 1}+c_{I}^{\prime} \rho_{b^{\prime} 1, b^{\prime} 1}+c_{I I}^{\prime} \rho_{c^{\prime} 1, c^{\prime} 1}
\end{aligned}
$$

for the populations and for the coherences

$$
\begin{aligned}
& \dot{\rho}_{c^{\prime} 0, a^{\prime} 1}=-\left(i \omega_{I I}^{\prime}-\omega_{2}+\frac{\gamma_{2}+c_{I I}^{\prime}}{2}\right) \rho_{c^{\prime} 0, a^{\prime} 1}+c_{c r, I}^{\prime} \rho_{d^{\prime} 0, b^{\prime} 1}, \\
& \dot{\rho}_{d^{\prime} 0, b^{\prime} 1}=-\left(i \omega_{I I}^{\prime}+\omega_{2}+\frac{\gamma_{2}+2 c_{I}^{\prime}+c_{I I}^{\prime}}{2}\right) \rho_{d^{\prime} 0, b^{\prime} 1}, \\
& \dot{\rho}_{b^{\prime} 0, a^{\prime} 1}=-\left(i \omega_{I}^{\prime}+\omega_{2}+\frac{\gamma_{2}+c_{I}^{\prime}}{2}\right) \rho_{b^{\prime} 0, a^{\prime} 1}+c_{c r, I I}^{\prime} \rho_{d^{\prime} 0, c^{\prime} 1}, \\
& \dot{\rho}_{d^{\prime} 0, c^{\prime} 1}=-\left(i \omega_{I}^{\prime}+\omega_{2}+\frac{\gamma_{2}+c_{I}^{\prime}+2 c_{I I}^{\prime}}{2}\right) \rho_{d^{\prime} 0, c^{\prime} 1}, \\
& \dot{\rho}_{b^{\prime} 0, c^{\prime} 0}=\left(i \omega_{c^{\prime} b^{\prime}}-\frac{c_{I}^{\prime}+c_{I I}^{\prime}}{2}\right) \rho_{b^{\prime} 0, c^{\prime} 0}+\gamma_{2} \rho_{b^{\prime} 1, c^{\prime} 1}, \\
& \dot{\rho}_{b^{\prime} 1, c^{\prime} 1}=\left(i \omega_{c^{\prime} b^{\prime}}-\gamma_{2}-\frac{c_{I}^{\prime}+c_{I I}^{\prime}}{2}\right) \rho_{b^{\prime} 1, c^{\prime} 1},
\end{aligned}
$$


Dissipative effects on a scheme of generation of a $W$ state in an array of coupled Josephson junctions 11

where $\omega_{c^{\prime} b^{\prime}}=E_{c}^{\prime}-E_{b}^{\prime}$. Also in this case, it is possible to verify that the solutions of these differential equations are given by

$$
\begin{aligned}
& \rho_{a^{\prime} 1, a^{\prime} 1}(t)=\rho_{a^{\prime} 1, a^{\prime} 1}\left(\tau_{1}\right) \mathrm{e}^{-\gamma_{2}\left(t-\tau_{1}\right)}, \\
& \rho_{c^{\prime} 0, c^{\prime} 0}(t)=\rho_{c^{\prime} 0, c^{\prime} 0}\left(\tau_{1}\right) \mathrm{e}^{-c_{I I}^{\prime}\left(t-\tau_{1}\right)}, \\
& \rho_{b^{\prime} 0, b^{\prime} 0}(t)=\rho_{b^{\prime} 0, b^{\prime} 0}\left(\tau_{1}\right) \mathrm{e}^{-c_{I}^{\prime}\left(t-\tau_{1}\right)}, \\
& \rho_{a^{\prime} 0, a^{\prime} 0}(t)=1-\rho_{b^{\prime} 0, b^{\prime} 0}\left(\tau_{1}\right) \mathrm{e}^{c_{I}^{\prime}\left(t-\tau_{1}\right)}-\rho_{c^{\prime} 0, c^{\prime} 0}(0) \mathrm{e}^{c_{I I}^{\prime}\left(t-\tau_{1}\right)} \\
& -\rho_{a^{\prime} 1, a^{\prime} 1}\left(\tau_{1}\right) \mathrm{e}^{-\gamma_{2}\left(t-\tau_{1}\right)} \\
& \rho_{c^{\prime} 0, a^{\prime} 1}(t)=\rho_{c^{\prime} 0, a^{\prime} 1}\left(\tau_{1}\right) \mathrm{e}^{\left(-i \omega_{I I}^{\prime}+i \omega_{2}-\frac{\gamma_{2}+c_{I I}^{\prime}}{2}\right)\left(t-\tau_{1}\right)}, \\
& \rho_{b^{\prime} 0, a^{\prime} 1}(t)=\rho_{b^{\prime} 0, a^{\prime} 1}\left(\tau_{1}\right) \mathrm{e}^{\left(-i \omega_{I}^{\prime}+i \omega_{2}-\frac{\gamma_{2}+c_{I}^{\prime}}{2}\right)\left(t-\tau_{1}\right)}, \\
& \rho_{b^{\prime} 0, c^{\prime} 0}(t)=\rho_{b 0, c 0}\left(\tau_{1}\right) \mathrm{e}^{\left(i \omega_{c^{\prime} b^{\prime}}-\frac{c_{I}^{\prime}+c_{I I}^{\prime}}{2}\right)\left(t-\tau_{1}\right)} .
\end{aligned}
$$

At this point it is convenient to write the density matrix describing the system during the second step, i.e. at a generic instant of time $t>\tau_{1}$ in the basis of the qubits eigenstates $\{|k l m\rangle\} \equiv\left\{|k\rangle_{1} \otimes|l\rangle_{2} \otimes|m\rangle_{3}\right\}$ with $k, l, m=0,1$ :

$$
\begin{aligned}
\rho(t) & =\rho_{000,000}(t)|000\rangle\left\langle 000\left|+\rho_{100,100}(t)\right| 100\right\rangle\left\langle 100\left|+\rho_{010,010}(t)\right| 010\right\rangle\langle 010| \\
& +\rho_{001,001}(t)|001\rangle\left\langle 001\left|+\rho_{100,010}(t)\right| 100\right\rangle\left\langle 010\left|+\rho_{100,010}^{*}(t)\right| 010\right\rangle\langle 100| \\
& +\rho_{100,001}(t)|100\rangle\left\langle 001\left|+\rho_{100,001}^{*}(t)\right| 001\right\rangle\left\langle 100\left|+\rho_{001,010}(t)\right| 001\right\rangle\langle 010| \\
& +\rho_{001,010}^{*}(t)|010\rangle\langle 001|,
\end{aligned}
$$

where

$$
\begin{aligned}
\rho_{000,000}(t) & =\rho_{a^{\prime} 0, a^{\prime} 0}(t), \\
\rho_{100,100}(t) & =\rho_{b^{\prime} 0, b^{\prime} 0}(t) \cos ^{2} \frac{\theta^{\prime}}{2}+\rho_{c^{\prime} 0, c^{\prime} 0}(t) \sin ^{2} \frac{\theta^{\prime}}{2} \\
& +2 \operatorname{Re}\left[\rho_{b^{\prime} 0, c^{\prime} 0}(t)\right] \sin \frac{\theta^{\prime}}{2} \cos \frac{\theta^{\prime}}{2}, \\
\rho_{010,010}(t) & =\rho_{a^{\prime} 1, a^{\prime} 1}(t), \\
\rho_{001,001}(t) & =\rho_{b^{\prime} 0, b^{\prime} 0}(t) \sin ^{2} \frac{\theta^{\prime}}{2}+\rho_{c^{\prime} 0, c^{\prime} 0}(t) \cos ^{2} \frac{\theta^{\prime}}{2} \\
& -2 \operatorname{Re}\left[\rho_{b^{\prime} 0, c^{\prime} 0}(t)\right] \sin \frac{\theta^{\prime}}{2} \cos \frac{\theta^{\prime}}{2}, \\
\rho_{100,010}(t) & =\rho_{b^{\prime} 0, a^{\prime} 1}(t) \cos \frac{\theta^{\prime}}{2}+\rho_{c^{\prime} 0, a^{\prime} 1}(t) \sin \frac{\theta^{\prime}}{2},
\end{aligned}
$$




$$
\begin{aligned}
\rho_{100,001}(t) & =\left[-\rho_{b^{\prime} 0, b^{\prime} 0}(t)+\rho_{c^{\prime} 0, c^{\prime} 0}(t)\right] \sin \frac{\theta^{\prime}}{2} \cos \frac{\theta^{\prime}}{2} \\
& +\rho_{b^{\prime} 0, c^{\prime} 0}(t) \cos ^{2} \frac{\theta^{\prime}}{2}-\rho_{b^{\prime} 0, c^{\prime} 0}^{*}(t) \sin ^{2} \frac{\theta^{\prime}}{2} \\
\rho_{001,010}(t) & =-\rho_{b^{\prime} 0, a^{\prime} 1}(t) \sin \frac{\theta^{\prime}}{2}+\rho_{c^{\prime} 0, a^{\prime} 1}(t) \cos \frac{\theta^{\prime}}{2} .
\end{aligned}
$$

From Eqs. (55)-(62) one can obtain all the information about the effects of dissipation on the system dynamics.

\section{The system dynamics: microscopic and phenomenological approaches}

Exploiting the results obtained in the previous section, we now analyze the effects of the reservoirs on the generation of the $\mathrm{W}$ state given in Eq. (1). In what follows we assume $g_{M 2}=g_{M 3}$ and $\omega_{M}=\omega_{2}=\omega_{3}=10 g_{M 2}$, which are typical values for the current superconducting technology [30].

The scope of the analysis will be twofold. On one hand we want to point out how the losses affect the scheme for the generation of the $\mathrm{W}$ state. On the other hand, we want to compare the predictions of our model given by Eqs. (4)-(5) with those predicted by the phenomenological model in Eq. (23), in order to understand the limits of validity of the latter. We calculate the fidelity $\mathcal{F}=\left\langle W\left|\rho\left(\tau_{1}, \tau_{2}\right)\right| W\right\rangle$ of the density matrix of the system at the time $t=\tau_{1}+\tau_{2}$ with respect to the target state $|W\rangle$ in Eq. (1). Figure 2 displays the time dependence of this function with respect to the durations $\tau_{1}$ and $\tau_{2}$ of the two steps of the generation scheme. The maximum of the fidelity, which is around 0.76 , is calculated by considering decay rates of the order of $0.1 g_{M 2}$ and corresponds to a choice of times very close to that in Ref. [19], i.e., $\bar{\tau}_{1}=2 g_{M 2}^{-1} \arcsin (1 / \sqrt{3})$ and $\bar{\tau}_{2}=2 g_{M 3}^{-1} \arccos (1 / \sqrt{2})$. As foreseeable the interaction of each qubit with the reservoir significantly affects the $\mathrm{W}$-state generation scheme. For this reason, even if for some purposes a value of $75 \%$ can be reasonable, it can be of interest to identify the state reached at the time instants considered. A reasonable guess would be to assume that, at the time instants $\bar{\tau}_{1}=2 g_{M 2}^{-1} \arcsin (1 / \sqrt{3})$ and $\bar{\tau}_{2}=2 g_{M 3}^{-1} \arccos (1 / \sqrt{2})$ the state is described by a Werner-like state $\rho_{W}=p|000\rangle\langle 000|+(1-p)| W\rangle\langle W|$, where $p$ is the (time-dependent) probability that at $t=\bar{\tau}_{1}+\bar{\tau}_{2}$ the system is found in its ground state $|000\rangle[31]$. In order to verify that this is indeed the case, we calculate the fidelity $F\left(\bar{\tau}_{1}, \bar{\tau}_{2}\right)=\operatorname{Tr}\left(\sqrt{\sqrt{\rho_{W}} \rho\left(\bar{\tau}_{1}, \bar{\tau}_{2}\right) \sqrt{\rho_{W}}}\right)$, finding that it is about 0.98 . We may thus conclude that, when dissipative effects are not negligible, the step-by-step scheme described in Ref. [19] can be exploited to generate the Werner-like state $\rho_{W}$ instead of the W state defined in Eq. (1).

Since one can wonder how the predictions change if one uses the phenomenological model to describe losses, let us now compare the predictions of the microscopic model just analyzed and the numerical solution of the phenomenological master equation (23). A good measure of the discrepancy between the predictions of the two models is the 


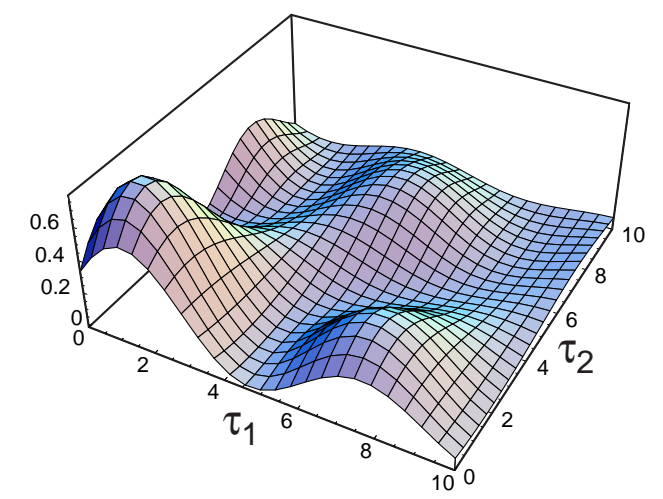

Figure 2. Dependence of the fidelity $\mathcal{F}$ on the durations of the two steps $\tau_{1}$ and $\tau_{2}$. Here $\omega_{M}=\omega_{2}=\omega_{3}=10 g_{M 2}$, the decay rates are of the order of $0.1 g_{M 2}$ and the times are in units of $g_{M 2}^{-1}=g_{M 3}^{-1}$.

trace distance [32]

$$
D\left(\rho_{\text {mic }}, \rho_{\text {phen }}\right)=\frac{1}{2} \operatorname{Tr}\left(\left|\rho_{\text {mic }}-\rho_{\text {phen }}\right|\right),
$$

which quantifies the imbalance between the probability distributions described by the states $\rho_{\text {mic }}$, predicted by the microscopic model, and $\rho_{\text {phen }}$, predicted by the phenomenological one.

We choose the same Lorentzian shape for the spectra of the three reservoirs

$$
J(\omega)=\frac{\gamma_{0} \lambda^{2}}{\left(\omega-\omega_{0}\right)^{2}+\lambda^{2}}
$$

with $\omega_{0}=\omega_{M}=\omega_{2}=\omega_{3}$. For very large values of the width $\lambda$ one recovers the results for flat spectrum and the decay rates are all equal to the qubit-reservoir coupling constant $\gamma_{0}$. We calculate the trace distance (63) for two cases: a) $\lambda=1000 g_{M 2}$ and $\gamma_{0}=0.1 g_{M 2}$, which corresponds to losses with flat spectrum; b) $\lambda=0.01 g_{M 2}$ and $\gamma_{0}=0.1 g_{M 2}$, which corresponds to losses for a very structured reservoir. Numerical simulations of the phenomenological model in the first case (not shown in figures), clearly demonstrate that, when the spectra of the reservoirs are equal and flat, the distance between the two states is very small. Therefore the effect of losses are essentially the same for both models, so that we may claim that the microscopically derived master equation is equivalent to the phenomenological one in such a case. A similar conclusion was previously found in the context of cavity-QED, where a phenomenological model of cavity losses, with the dissipative part of the master equation depending only on the single-mode operators, turns out to be microscopically justifiable in the case of flat reservoir spectrum [33, 34].

In the second case of structured spectrum, instead, the values of the decay rates are very sensitive to the Bohr frequencies at which they are evaluated. As a consequence, as shown in Fig. 3, the trace distance between the states predicted by the phenomenological and microscopic models can reach very large values. In order to understand the origin of the large distance between the states predicted in this last case, one can look at 


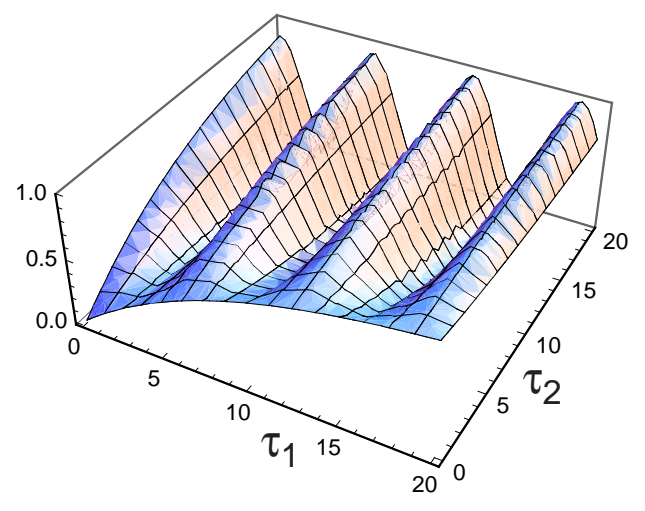

Figure 3. Distance between the microscopically and phenomenologically derived states for $\lambda=0.01 g_{M 2}$ and $\gamma_{0}=0.1 g_{M 2}$ as a function of $\tau_{1}$ and $\tau_{2}$. The times are in units of $g_{M 2}^{-1}=g_{M 3}^{-1}$.

the dynamical behavior of the populations of the states of the system predicted by the two models. Figures 4 (a), (b) and (c) show, as a function of the interaction times $\tau_{1}$ and $\tau_{2}$, the populations of the states $|000\rangle,|010\rangle$ and $|001\rangle$, correspondingly, calculated exploiting the phenomenological (top) and the microscopic (bottom) models.

From Fig. 4(a) it is clear that, while in the phenomenological model (top), all the excitations decay exponentially with the same rate with respect to both $\tau_{1}$ and $\tau_{2}$, in the microscopic model (bottom) the role of the two interaction times is very asymmetric: indeed, by varying the duration $\tau_{1}$ of the first step, one obtains initial states for the second step characterized by very different decay rates, so that some excitation is trapped or not in the system according to the value of $\tau_{1}$.

Consider now the decay properties of the population of the state $|010\rangle$ for the the phenomenological and microscopic models. Figure 4(b) (bottom) in particular shows that, depending on the choice of $\tau_{1}$, the initial condition of the second step oscillates with almost no damping, and then the state decays with the single-qubit decay rate.

As far as the decay properties of the state $|001\rangle$ are concerned, Figs. 4 (c) show that, while in the phenomenological model the oscillations between qubits $\mathrm{M}$ and 3 decay rapidly, in the microscopic model the oscillations are conserved for much longer times: this is due to the fact that in the microscopic model the decay rates are proportional to the value of the spectrum at the Bohr frequencies between dressed states, as one can see from Eqs. (13)-(12) and (15)-(20), and therefore, for the spectrum chosen in our example, they are much smaller than the decay rates in the phenomenological model, which are proportional to the value of the spectrum at the single-qubit frequencies, i.e., to its maximum. More in detail, for the spectrum and the value of $\lambda$ we are considering here (namely $\lambda=0.01 g_{M 2}$ ), the single qubit decay rate is equal to $\gamma_{0}=0.1 g_{M 2}$, while it is straightforward to show that the decay rates of the states involving the second qubit in the first step are equal to $J\left(\omega_{I}\right) \simeq J\left(\omega_{I I}\right) \simeq 0.4 \cdot 10^{-4} g_{M 2}$. The very small decay rates for the states involving the second qubit in the first step give rise to phenomena of population trapping, whose consequence is the conservation of the 

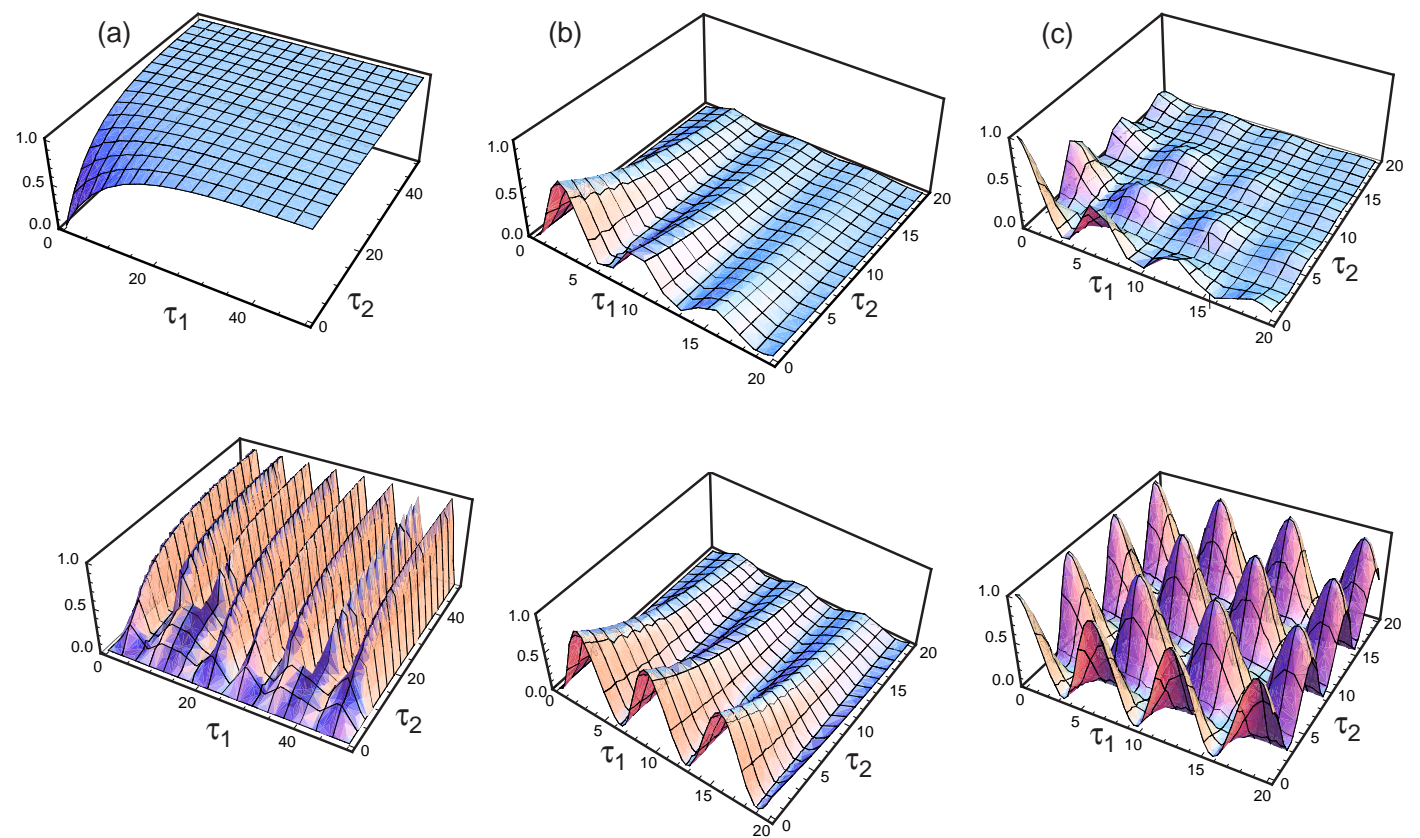

Figure 4. Populations of states (a) $|000\rangle$, (b) $|010\rangle$ and (c) $|100\rangle$ calculated by the phenomenological (top) and microscopic (bottom) models with $\lambda=0.01 g_{M 2}$ and $\gamma_{0}=0.1 g_{M 2}$, as functions of the interaction times $\tau_{1}$ and $\tau_{2}$ (in units of $g_{M 2}^{-1}=g_{M 3}^{-1}$ ).

population oscillations with respect to the interaction time $\tau_{1}$ in Fig. 4(b) (bottom). This point is analogous to what was previously found during the study of a microscopic non-Markovian model for cavity losses in cavity-QED [35].

\section{Conclusions}

In this paper, we have analyzed the effects of losses on a scheme of generation of multipartite entanglement. At the time instants of interest, the fidelity between the actual state of the system and the target state $|W\rangle$ is about $75 \%$. The deviation from unity can simply be understood as due to the fact that the system has decayed to the ground state $|000\rangle$. Thus, the scheme becomes a protocol aimed at the generation of a Werner-like state $\rho_{W}=P|000\rangle\langle 000|+(1-P)| W\rangle\langle W|$. We wish to underline that this statement is true when we consider a flat spectrum at $T=0 \mathrm{~K}$.

Moreover, the comparison between our microscopic model and a naiver but more popular phenomenological master equation has given us an occasion to understand the limit of a phenomenological approach in the context of our problem. In fact, we found that the predictions of the phenomenological and the microscopic approaches do not differ much when the spectra of the reservoirs are flat. However, when the reservoirs are structured the two models predict very different results, and in the microscopic model, the population trapping phenomena can occur, due to the very different values of the various decay rates involved, which cannot be predicted by the phenomenological master equation. This is an example in which the phenomenological model can lead to 
very different phenomena from those predicted by the master equation derived from the microscopic Hamiltonian.

Of course, at this stage of our study of structured reservoirs, we have only considered effects which occur at times much longer than the correlation times of the reservoirs. When this is not the case, a more complete non-Markovian theory must be adopted, for instance by means of a time-convolutionless master equation. In this case, the various decay rates would be time dependent, and would become stationary, and proportional to the value of the spectra at the relevant Bohr frequencies, only at times much longer than the memory times of the baths. This issue, as well as the study of other sources of noise typical of Josephson junctions, such as $1 / f$ and single impurity noise, will be the subject of future work.

\section{Acknowledgements}

This work is partly supported by the bilateral Italian-Japanese Project II04C1AF4E. Support from MIUR Projects No. II04C0E3F3 and PRIN 2008 (2008C3J43_003) is also acknowledged.

\section{Appendix A.}

In this Appendix, we list the eigenstates and eigenvalues of the Hamiltonians (2) and (3), corresponding to the first and second steps, respectively. Both sets of eigenstates and eigenvalues can be obtained by a straightforward diagonalization of the Hamiltonians.

The eigenstates of the Hamiltonian (2) are $|a\rangle \otimes|k\rangle_{3},|b\rangle \otimes|k\rangle_{3},|c\rangle \otimes|k\rangle_{3}$ and $|d\rangle \otimes|k\rangle_{3}$, where $k=0,1$ and

$$
\begin{aligned}
& |a\rangle=\left|0_{M} 0_{2}\right\rangle, \\
& |b\rangle=\cos \frac{\theta}{2}\left|1_{M} 0_{2}\right\rangle-\sin \frac{\theta}{2}\left|0_{M} 1_{2}\right\rangle, \\
& |c\rangle=\sin \frac{\theta}{2}\left|1_{M} 0_{2}\right\rangle+\cos \frac{\theta}{2}\left|0_{M} 1_{2}\right\rangle, \\
& |d\rangle=\left|1_{M} 1_{2}\right\rangle,
\end{aligned}
$$

with

$$
\sin \theta=\frac{\left|g_{M 2}\right|}{\sqrt{\left(\omega_{2}-\omega_{M}\right)^{2}+g_{M 2}^{2}}}, \quad \cos \theta=\frac{\left|\omega_{2}-\omega_{M}\right|}{\sqrt{\left(\omega_{2}-\omega_{M}\right)^{2}+g_{M 2}^{2}}} .
$$

The corresponding eigenvalues are

$$
\begin{aligned}
& E_{a}=0, \\
& E_{b}=\frac{1}{2}\left(\omega_{M}+\omega_{2}\right)-\frac{1}{2} \sqrt{\left(\omega_{2}-\omega_{M}\right)^{2}+g_{M 2}^{2}}, \\
& E_{c}=\frac{1}{2}\left(\omega_{M}+\omega_{2}\right)+\frac{1}{2} \sqrt{\left(\omega_{2}-\omega_{M}\right)^{2}+g_{M 2}^{2}}, \\
& E_{d}=\omega_{M}+\omega_{2} .
\end{aligned}
$$


Similarly, the eigenstates of the Hamiltonian (3) are expressed as $\left|a^{\prime}\right\rangle \otimes|k\rangle_{2}$, $\left|b^{\prime}\right\rangle \otimes|k\rangle_{2},\left|c^{\prime}\right\rangle \otimes|k\rangle_{2}$ and $\left|d^{\prime}\right\rangle \otimes|k\rangle_{2}$, where $k=0,1$ and

$$
\begin{aligned}
\left|a^{\prime}\right\rangle & =\left|0_{M} 0_{3}\right\rangle, \\
\left|b^{\prime}\right\rangle & =\cos \frac{\theta^{\prime}}{2}\left|1_{M} 0_{3}\right\rangle-\sin \frac{\theta^{\prime}}{2}\left|0_{M} 1_{3}\right\rangle, \\
\left|c^{\prime}\right\rangle & =\sin \frac{\theta^{\prime}}{2}\left|1_{M} 0_{3}\right\rangle+\cos \frac{\theta^{\prime}}{2}\left|0_{M} 1_{3}\right\rangle, \\
\left|d^{\prime}\right\rangle & =\left|1_{M} 1_{3}\right\rangle,
\end{aligned}
$$

with

$$
\sin \theta^{\prime}=\frac{\left|g_{M 3}\right|}{\sqrt{\left(\omega_{3}-\omega_{M}\right)^{2}+g_{M 3}^{2}}}, \quad \cos \theta^{\prime}=\frac{\left|\omega_{3}-\omega_{M}\right|}{\sqrt{\left(\omega_{3}-\omega_{M}\right)^{2}+g_{M 3}^{2}}} .
$$

The corresponding eigenvalues are

$$
\begin{aligned}
& E_{a^{\prime}}=0, \\
& E_{b^{\prime}}=\frac{1}{2}\left(\omega_{M}+\omega_{3}\right)-\frac{1}{2} \sqrt{\left(\omega_{3}-\omega_{M}\right)^{2}+g_{M 3}^{2}}, \\
& E_{c^{\prime}}=\frac{1}{2}\left(\omega_{M}+\omega_{3}\right)+\frac{1}{2} \sqrt{\left(\omega_{3}-\omega_{M}\right)^{2}+g_{M 3}^{2}}, \\
& E_{d^{\prime}}=\omega_{M}+\omega_{3} .
\end{aligned}
$$

\section{References}

[1] Vion D., Aassime A., Cottet A., Joyez P., Pothier H., Urbina C., Esteve D., and Devoret M. H., (2002) Science 296, 886.

[2] Martinis J. M., Nam S., Aumentado J., and Urbina C., (2002) Phys. Rev. Lett. 89, 117901.

[3] Pashkin Yu. A., Yamamoto T., Astafiev O., Nakamura Y., Averin D. V., and Tsai J. S., (2003) Nature 421, 823 .

[4] Chiorescu I., Nakamura Y., Harmans C. J. P. M., and Mooij J. E., (2003) Science 299, 1896.

[5] Wallraff A., Schuster D. I., Blais A., Frunzio L., Huang R. S., Majer J., Kumar S., Girvin S. M., and Schoelkopf R. J., (2004) Nature 431, 162.

[6] Lucero E., Hofheinz M., Ansmann M., Bialczak R. C., Katz N., Neeley M., OConnell A. D., Wang H., Cleland A. N., Martinis J. M., (2008) Phys. Rev. Lett. 100, 247001.

[7] Chow J. M., Gambetta J. M., Tornberg L., Koch J., Bishop L. S., Houck A. A., Johnson B. R., Frunzio L., Girvin S. M., Schoelkopf R. J., (2009) Phys. Rev. Lett. 102, 090502.

[8] Clarke J. and Wilhelm F. K., (2008) Nature 453, 1031.

[9] Schreier J. A., Houck A. A., Koch J., Schuster D. I., B. R. Johnson, Chow J. M., Gambetta J. M., Majer J., Frunzio L., Devoret M. H., Girvin S. M., Schoelkopf R. J., (2008) Phys. Rev. B 77, 180502(R).

[10] Yamamoto T., Pashkin Yu. A., Astfiev O., and Nakamura Y., (2003) Nature 425, 941.

[11] McDermott R., Simmonds R. W., Steffen M., Cooper K. B., Cicak K., Osborn K. D., Oh S., Pappas D. P., and Martinis J. M., (2005) Science 307, 1299.

[12] Hime T., Reichardt P. A., Plourde B. L. T., Robertson T. L., Wu C.-E., Ustinov A. V., Clarke J., (2006) Science 314, 1427.

[13] Plantenberg J. H., de Groot P. C., Harmans C. J. P. M., and Mooij J. E., (2007) Nature 447, 836.

[14] Niskanen A. O., Harrabi K., Yoshihara F., Nakamura Y., Lloyd S., Tsai J. S., (2007) Science 316, 723. 
[15] Siddiqi I., Vijay R., Pierre F., Wilson C.M., Metcalfe M., Rigetti C., Frunzio L., Devoret M. H., (2004) Phys. Rev. Lett. 93, 207002.

[16] McDermott R., Simmonds R. W., Steffen M., Cooper K. B., Cicak K., Osborn K. D., Oh S., Pappas D. P., Martinis J. M., (2005) Science 307, 1299.

[17] Steffen M., Ansmann M., Bialczak R. C., Katz N., Lucero E., McDermott R., Neeley M., Weig E. M., Cleland A. N., Martinis J. M., (2006) Science 313, 1423.

[18] Wei L. F., Liu Y. X., and Nori F., Phys. Rev. Lett. 96, 246803 (2006); Matsuo S., Ashhab S., Fujii T., Nori F., Nagai K., Hatakenaka N., (2007) J. Phys. Soc. Jpn. 76, 054802; Galiautdinov A. and Martinis J. M., (2008) Phys. Rev. A 78, 010305.

[19] Migliore R., Yuasa K., Nakazato H., and Messina A., (2006) Phys. Rev. B 74, 104503.

[20] Migliore R., Yuasa K., Guccione M., Nakazato H., and. Messina A, (2007) Phys. Rev. B 76, 052501.

[21] Altomare F., Park J.I., Cicak K., Sillanp M. A., Allman MS., Li D., Sirois A., Strong J.A., Whittaker J.D., Simmonds R.W., (2010) Nature Physics 6, 777.

[22] Neeley M., Bialczak R. C., Lenander M., Lucero E., Mariantoni M., O’Connell A. D., Sank D., Wang H., Weides M., Wenner J., Yin Y., Yamamoto T., Cleland A. N., Martinis J. M., (2010) Nature 467, 570 .

[23] DiCarlo L., Reed M. D., Sun L., Johnson B. R., Chow J. M., Gambetta J. M., Frunzio L., Girvin S. M., Devoret M. H., Schoelkopf R. J., (2010) Nature 467, 574.

[24] Scala M., Migliore R., and Messina A., (2008) J. Phys. A: Math. Theor. 41, 435304.

[25] Migliore R., Scala M., Guccione M., Sánchez-Soto L. L., Messina A., (2009) Phys. Status Solidi B 246, 1013.

[26] Scala M., Migliore R., Messina A., and Sanchez-Soto L.L., in press on European Physical Journal D (2011), published onlne on http://dx.doi.org/10.1140/epjd/e2010-00230-5

[27] Mazzola L., Maniscalco S., Piilo J., Suominen K.-A., and Garraway B. M., (2009) Phys. Rev. A 80, 012104; Ferraro E., Scala M., Migliore R., and Napoli A., (2009) Phys. Rev. A 80, 042112; Sinayskiy I., Ferraro E., Napoli A., Messina A., and Petruccione F., (2009) J. Phys. A: Math. Theor. 42, 485301; Yu T. and Eberly J. H., (2010) Opt. Commun. 283, 676.

[28] Castellano M. G., Chiarello F., Leoni R., Simeone D., Torrioli G., Cosmelli C., and Carelli P., (2005) Appl. Phys. Lett. 86, 152504.

[29] Breuer H.-P. and Petruccione F. 2002 The Theory of Open Quantum Systems (Oxford: Oxford University Press)

[30] You J. Q., Nori F., (2005) Phys. Today 58, 42; Devoret M. H., Martinis J., (2004) Quantum Inf. Process. 3, 163.

[31] Werner R. F., (1989) Phys. Rev. A 40, 4277; Munro W. J., James D. F. V., White A. G., and Kwiat P. G., (2001) Phys. Rev. A 64, 030302(R); Wei T. C. et al., (2003) Phys. Rev. A 67, 022110.

[32] Nielsen M. A. and Chuang I. L., Quantum Computation and Quantum Information (Cambridge University Press, Cambridge, 2000).

[33] Scala M., Militello B., Messina A., Piilo J., and Maniscalco S., (2007) Phys. Rev. A 75, 013811.

[34] Scala M., Militello B., Messina A., Maniscalco S., Piilo J., and Suominen K.-A., (2007) J. Phys. A: Math. Theor. 40, 14527.

[35] Scala M., Militello B., Messina A., Maniscalco S., Piilo J., and Suominen K.-A., (2008) Phys. Rev. A 77, 043827. 